Results During the study period 56 elderly patients were admitted to the Stroke Unit. At the time of admission 63 prescribing omissions were found in $69.8 \%$ of elderly (average 1.19 omissions per patient), of which $74.5 \%(n=38)$ were corrected at the time of discharge. Prescribing omissions were also detected in $80.9 \%$ of patients receiving five or more medications simultaneously. In 10 patients, 13 omissions found at admission were not corrected during hospitalisation, and in three patients three new omissions were detected.

Conclusion The prevalence of prescribing omissions of cardiovascular risk management therapy in elderly patients admitted to a Stroke Unity is high. START criteria is an evidence-based and easy-to-use screening tool that can assist clinicians in the optimisation of geriatric therapy, particularly in relation to cardiovascular disease prevention.

\section{SP2-8 BIAS REDUCTION AND PRECISION IN DIFFERENT TYPES OF CONTROL SELECTION IN ANALYTICAL CROSS-SECTIONAL STUDIES; A METHODOLOGICAL PAPER}

doi:10.1136/jech.2011.142976n.98

\begin{abstract}
${ }^{1,2}$ A Mirzazadeh, ${ }^{*}$ A Ahmadvad, ${ }^{1,3} \mathrm{~J}$ Gholami, ${ }^{1} \mathrm{~A}$ Mohammadpour. 'Department of Biostatistics and Epidemiology, School of public health, Tehran University of Medical Sciences, Tehran, Iran; ${ }^{2}$ Regional Knowledge Hub for HIVIAIDS Surveillance, Kerman University of Medical Sciences, Kerman, Iran, ${ }^{3}$ Knowledge Utilisation Research Center, Tehran University of Medical Sciences, Tehran, Iran
\end{abstract}

Introduction Control selection is a crucial step at the study designing phase. Although, the concept of the different types of matching for control selection has been discussed in the context of case-control studies, here we targeted analytical cross-sectional studies to explore the effects of each type of control selection on the amount of bias and precision of the OR.

Methods 41 coronary atherosclerotic patients and 92 disease-free hospital controls were recruited to assess the relationship between opium consumption (OpiumHx) and coronary atherosclerosis (Outcome). Considering the OpiumHx as the main independent factor and age as the confounder, we calculated point estimate and the CI for OR in different scenarios of matching for control selection, namely exact, stratified, frequency and propensity matching. Syntaxes were developed by STATA 10.

Results The crude OR was 3.4 (95\% CI 1.5 to 7.9). By exact matching on age, 21 pairs remained for the analysis and the OR was equal to 3.3 (0.8 to 18.8). Stratified matching on age group kept 41 pairs and gave us the OR of 0.9 (0.5 to 1.7). Frequency matching kept 88 subjects for the analysis and led to the OR of 3.0 (1.2 to 7.4). By propensity matching, 27 pairs remained which gave the OR of 3.5 (1.1 to 14.6).

Conclusion Matching techniques influence effect size and precision, seriously. Although the most bias reduction happened in pair matched techniques, a large reservoir of controls would be needed to prohibit immense decrease in precision. These findings should be considered at both protocol development and analysis phases of observational studies with caution.

\section{SP2-9 DIAGNOSTIC CRITERIA OF LIPODYSTROPHY IN HIV- INFECTED PATIENTS}

doi:10.1136/jech.2011.142976n.99

\footnotetext{
${ }^{1,2} \mathrm{P}$ R de Alencastro, ${ }^{* 3,4} \mathrm{~S}$ C Fuchs, ${ }^{3,5} \mathrm{~F}$ H Wolff, ${ }^{2,4} \mathrm{M}$ L R Ikeda, ${ }^{5,6} \mathrm{~A}$ B M Brandão, ${ }^{3,5} \mathrm{~N}$ T Barcellos. ${ }^{1}$ Postgraduate Program in Medicine: Medical Sciences, Universidade Federal do Rio Grande do Sul, Porto Alegre-RS, Brazil; ${ }^{2}$ Hospital Sanatório Partenon, State Department of Health, Rio Grande do Sul, Porto Alegre-RS, Brazil, ${ }^{3}$ Postgraduate Studies Program in Epidemiology, School of Medicine, Universidade Federal do Rio Grande do Sul, Porto Alegre-RS, Brazil; ${ }^{4}$ Postgraduate Studies Program in Cardiology, School of Medicine, Universidade Federal do Rio Grande do Sul, Porto Alegre-RS, Brazil; ${ }^{5}$ Ministry of Science and Technology and Office of Technology Assessment in
}

Health (IATS/CNPq), Hospital de Clinicas de Porto Alegre, Porto Alegre-RS, Brazil, ${ }^{6}$ Faculdade de Medicina, Universidade Federal de Ciências da Saúde de Porto Alegre, Porto Alegre-RS, Brazil

Introduction The prevalence of lipodystrophy ranges from 2 to $84 \%$ and the range of findings stems from differences between the populations studied and lack of standardised diagnostic criteria. The diagnosis of lipodystrophy is based on changes in body fat distribution with or without medical confirmation, objective measures of circumferences and skin folds or quantification of adiposity by dual emission x-ray absorptiometry (DEXA) CT scan or MRI.

Objective Establish diagnostic criteria for lipodystrophy and evaluate the prevalence of lipodystrophy among men and women with HIV/AIDS

Study design Cross-sectional survey was conducted in HIV-infected patients of both genders, aged 18 years or older who sought to confirm the diagnosis or treatment in a reference service for HIV/ AIDS for the period June 2006 to December 2008.

Results 1240 patients with HIV infection were invited to participate. Among the signs that contributed most to the detection of lipoatrophy, include hollow cheeks, reduced fat on the face, buttocks and arms. To lipohypertrophy the biggest contributor was an increase in fat in the abdomen, abdomen bigger than usual and increased waist circumference. Men were more often lipoatrophy $(p=0.049)$ and women lipohypertrophy $(p<0.001)$.

Conclusion This study identified high rates of self-reported signs of lipodystrophy were significantly associated with that objective measures. The differences between men and women do not represent a formal test validation, but the analysis comparing objective measures confirms the importance of using specific questions about changes in the distribution of fat in their accompaniment.

\section{SP2-10 METHODS FOR BUILDING DIFFERENT TYPES OF AREAS FOR DIFFERENT APPLICATIONS IN PUBLIC HEALTH}

doi:10.1136/jech.2011.142976n.100

${ }^{1} \mathrm{R}$ Pinheiro, ${ }^{*} \mathrm{E}$ Oliveira, ${ }^{3} \mathrm{E}$ Prates, ${ }^{4} \mathrm{M}$ Carvalho. ${ }^{1}$ Federal University of Rio de Janeiro, Rio de Janeiro, Brazil; ' ${ }^{2}$ Health Information Network Group, Rio de Janeiro, Brazil; ${ }^{3}$ Federal University of Rio de Janeiro State, Rio de Janeiro, Brazil; ${ }^{4}$ Osvaldo Cruz Institute, Rio de Janeiro, Brazil

Introduction Spatial distribution of health indicators and health services use are important for the evaluation of population health and managers actions. In urban areas, to build small areas is not trivial, because there are different travelling possibilities for treatment. The aim of this paper is to present methods for building different types of areas for different applications in public health in a big city in Brazil.

Metodology We used the (x,y) coordinates of health services and tuberculosis cases. The indicators of population characteristics were located at census tract centroids. Tuberculosis rates were mapped using the ratio between the spatial smoothing of tuberculosis cases and the spatial smoothing of population. Catchment area of a health service was mapped based on the spatial smoothing of the tuberculosis cases treated in this service. The health service market was built using the ratio between the spatial smoothing of the cases treated in this service and the spatial smoothing of all disease cases. For risk areas, we mapped the spatial smoothing for each population feature. We sum all maps to elaborate a resultant one.

Results Influence areas differed from health service markets. Health service regionalisation was only partially similar to coverage areas defined by the Tuberculosis Control Program. The tuberculosis rates and risk areas showed some correlation.

Conclusion Different area types shows different types of information for the diagnosis of health conditions, population and health service resources profile, indicating the relation between the population, the health services and the territory. 